\title{
THE EXCEPTIONAL CASE IN A THEOREM OF BOSE AND SHRIKHANDE
}

\author{
Dedicated to Professor G. Szekeres on his 65th birthday \\ PAUL de WITTE* \\ (Received 23 January. 1975; revised 9 January, 1976) \\ Communicated by Jennifer Seberry Wallis
}

\begin{abstract}
It is shown that ( $n$ being an integer) any non-trivial finite linear space with $n^{2}-1$ points. all of degree at most $n+1$, is embeddable in a finite projective plane of order $n$. This generalizes a theorem of Bose and Shrikhande and settles the unsolved case $n=6$.
\end{abstract}

\section{Introduction}

We shall follow the terminology and notation of several previous papers, mentioned in the reference list. Nevertheless, it may be useful to recall the meaning of a few important terms and symbols. By a finite linear space $(=F L S)$ is meant a finite set of $p$ so-called points together with a set of $q$ subsets of points, called lines, such that every pair of distinct points is included in precisely one line and every line contains at least two points. If there are at least two lines, the FLS is said to be non-trivial. Professor Szekeres was one of the first to show that $q \geqq p$ in any non-trivial FLS.

The number of points on a line $x$ will be denoted by $a(x)$ and called the degree of $x$. Similarly, the number of lines through a point $u$ will be denoted by $b(u)$ and called the degree of $u$. A $k$-line is a line of degree $k$, and a $k$-point is a point of degree $k$. If there is a line of degree $p-1$, the FLS is

\footnotetext{
* Partially supported by National Research Council of Canada Grant.
} 
called a near-pencil. If all lines are of degree two, the FLS is called a complete graph. Two lines are said to miss each other if they are disjoint; they are called parallel if they are equal or disjoint. Note that disjointness and parallelism need not be transitive.

Let us also mention that most of the time the points and lines of an FLS will be denoted by the symbols $u_{\alpha}(1 \leqq \alpha \leqq p)$ and $x_{\sigma}(1 \leqq \sigma \leqq q)$ respectively, introduced so that $\alpha \leqq \beta$ implies $b\left(u_{\alpha}\right) \geqq b\left(u_{\beta}\right)$ and $\sigma \leqq \tau$ implies $a\left(x_{\sigma}\right) \geqq a\left(x_{\tau}\right)$. For brevity's sake, we shall write $b_{\alpha}$ for $b\left(u_{\alpha}\right)$ and $a_{\sigma}$ for $a\left(x_{\sigma}\right)$. In accordance with this, the expression $b_{1} \leqq n+1$ will mean that there are no points of degree $>n+1$. The incidence number of a point $u_{\alpha}$ and a line $x_{\sigma}$, defined as 1 if $u_{\alpha}$ lies on $x_{\sigma}$ and as 0 if it does not, will be denoted by $r_{\sigma \alpha}$.

A finite projective plane $(=F P P)$ of order $n(\geqq 2)$ is nothing but an FLS such that $p=q=n^{2}+n+1$ and $a_{\sigma}=b_{\alpha}=n+1$ for all $x_{\sigma}$ and $u_{\alpha}$; and a finite affine plane $(=F A P)$ of order $n$ is an FLS such that $p=q-n=n^{2}$ and $a_{\sigma}=b_{\alpha}-1=n$ for all $x_{\sigma}$ and $u_{\alpha}$.

By an $s$-curve in an FPP of order $n$ will be meant any subset of $s$ points no three of which are collinear. It was shown by Bose (1947) that $s \leqq n+2$ and that $s=n+2$ can only occur if $n$ is even. Further basic properties were given by Qvist (1952).

By the complement of an $s$-curve is meant the FLS obtained by removing all its points from the FPP it is part of. It is not hard to show that the complement of an $s$-curve in an FPP of order $n$ satisfies the following conditions (assuming that $s \leqq 1$ if $n=2$ ):

(A) There are $p=n^{2}+n+1-s$ points, all of degree $n+1$.

(B) There are $q=n^{2}+n+1$ lines, of which $s(s-1) / 2$ are of degree $n-1$, $s(n+2-s)$ of degree $n$, and the remainder of degree $n+1$.

(C) If a point lies on $t$ lines of degree $n$, then it lies also on $n+1-(s+t) / 2$ lines of degree $n+1$ and on $(s-t) / 2$ lines of degree $n-1$. Hence $s \geqq t$ and $s \equiv t(\bmod 2)$.

It follows from (B) and (C) that $t=0$ if $s=n+2$. Consequently, the complement of an $(n+2)$-curve in an FPP of even order $n(\geqq 4)$ satisfies the following conditions:

(a) There are $p=n^{2}-1$ points, all of degree $n+1$.

(b) There are $q=n^{2}+n+1$ lines, of which $(n+2)(n+1) / 2$ are of degree $n-1$ and $n(n-1) / 2$ of degree $n+1$.

(c) Every point lies on $n / 2$ lines of degree $n+1$ and on $(n+2) / 2$ lines of degree $n-1$.

This leads to the question whether a sort of converse holds. More precisely, does the assumption that an FLS satisfies the conditions $(A)(B)(C)$ 
with $n \geqq 2$ imply that an FPP of order $n$ exists and that the FLS is embeddable in one? A positive answer in the case of (a) (b) (c) with $n \neq 6$ was found by Bose and Shrikhande (1973); see also p. 65 of Bose (1973). Remarkably enough, they had to apply a well-known theorem on the characterization of the line graphs of the complete graphs $K_{m}$, which occurs in Harary (1969) as Theorem 8.6, for $m \neq 8$. The main purpose of this paper is to settle the exceptional case $n=6$, which is rather intriguing in view of Tarry's theorem on the nonexistence of FPP's of order six. It will be shown that no FLS satisfies the conditions $(a)(b)(c)$ when $n=6$ (cf. Theorem 1 in Section 4). In fact, we shall also prove that these conditions suffer from a considerable degree of redundancy (cf. Corollaries 1 and 2 and Theorem 2 in Section 4). The corollaries do not involve much novel work, but Theorem 2 requires two particular cases, stated in Section 3 as P7 and P8, of the following embedding result: If $n$ is an integer $\geqq 2$ and $\mathscr{L}$ a non-trivial FLS such that $p \geqq n^{2}$ and $b_{1} \leqq n+1$, then $\mathscr{L}$ is embeddable in an FPP of order $n$. Note that P7 is nothing but the finite case of Kepler's extension of affine planes to projective planes, based on his celebrated invention of points "at infinity" lying on a line "at infinity".

An immediate consequence of Theorem 2 and the above embedding result is the

THEOREM: If $n$ is an integer $\geqq 2$ and $\mathscr{L}$ a non-trivial FLS such that $p \geqq n^{2}-1$ and $b_{1} \leqq n+1$, then $\mathscr{L}$ is embeddable in an FPP of order $n$.

This shows that a positive answer can always be given to the italicized question above, whatever the values of $n(\geqq 2), s, t$ in the superabundant conditions (A) (B) (C). In looser terms, a small amount of well-chosen information about the complement of an $s$-curve always allows the reconstruction of the $s$-curve itself.

The theorem just obtained also bears upon another problem of linear geometry, of which I only became aware after completing the first draft. It is well known that a non-trivial FLS with $b_{1} \leqq n+1$ satisfies $p \leqq n^{2}+n+1$ and that this maximum is reached only in the case of the triangle and of an FPP of order $n$. This suggests the question what the maximal number of points is when the integer $n(\geqq 6)$ is not the order of any FPP. Denoting it by $p_{0}(n)$, we see that the above theorem implies $p_{0}(n) \leqq n^{2}-2$. Both these results have already been obtained by Vanstone (1974) except when $n=6$. His proof is cast in the language of " $(r, \lambda)$-designs" or "regular pair-wise balanced designs". As to $n=6$, he only got $p_{0}(6) \leqq n^{2}-1=35$. But he also observed that $p_{0}(6) \geqq 31$ in view of the existence of an FPP of order five, and he showed by a long calculation that $p_{0}(6) \neq 33,34$. The main new contribution of the 
present paper to this problem is therefore the result $p_{0}(6) \neq 35$, leaving us with only two conceivable values for $p_{0}(6)$.

It will be shown by rather elementary means (in Corollary 4 of Section 4 ) that ( $n$ being an integer) any FLS with $p \geqq n^{2}-1$ and $b_{1} \leqq n+1$ satisfies $q \leqq n^{2}+n+1$. That $n^{2}-1 \leqq p \leqq q \leqq n^{2}+n+1$ implies $b_{1} \leqq n+1$ in general, on the other hand, turns out to be a great deal more difficult. A substantial advance on the present results is therefore made in a forthcoming paper establishing the embeddability of most FLS's with $n^{2}-1 \leqq p \leqq q \leqq$ $n^{2}+n+1$.

I should like to thank Dr Scott Vanstone for having supplied me with a copy of his thesis, Dr Jim Totten and the referee for some useful suggestions, and my wife for her material and spiritual help.

\section{Prerequisites from graph theory}

We assume that all graphs are finite, without loops or multiple edges, but not necessarily nonempty. The characterization theorem referred to above may be stated as follows. Let $m$ be a natural number and $G$ a graph satisfying the four following conditions:

I. there are $m(m-1) / 2$ vertices;

II. every vertex has $2(m-2)$ neighbours;

III. every two adjacent vertices have $m-2$ common neighbours;

IV. every two non-adjacent vertices have four common neighbours.

Then $G$ is the line graph of the complete graph $K_{m}$ or one of three exceptional graphs, all with $m=8$.

This theorem is easy for $m \leqq 4$. It is due to W.S. Connor for $m \geqq 9$, to S. S. Shrikhande for $m=5,6$, to A. J. Hoffman for $m=7$, and to Chang Li-chien and A. J. Hoffman for $m=8$. For a complete proof, see Chang (1959, 1960). As Chang's papers are not easily available in general, descriptions of the three exceptional graphs may be of some use.

EXCEPTIONAL GRAPH I:

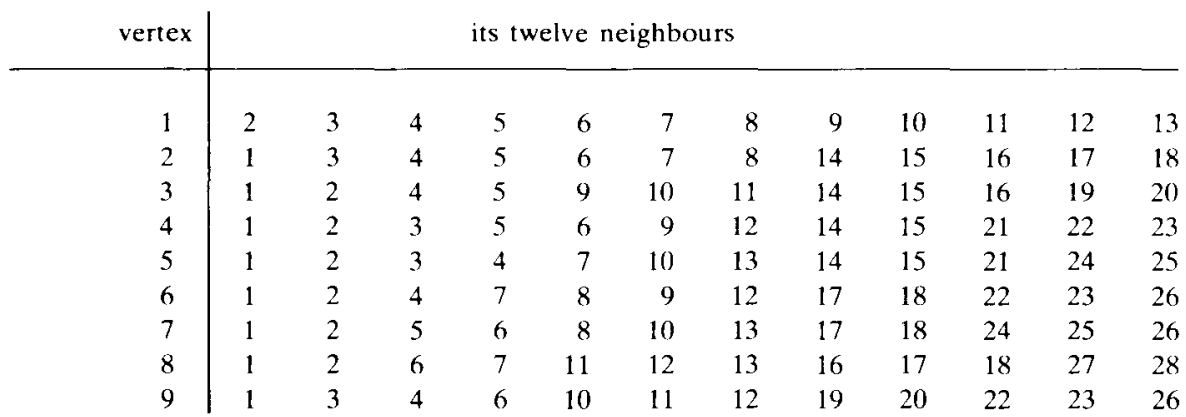




\begin{tabular}{l|rrrrrrrrrrrr}
10 & 1 & 3 & 5 & 7 & 9 & 11 & 13 & 19 & 20 & 24 & 25 & 26 \\
11 & 1 & 3 & 8 & 9 & 10 & 12 & 13 & 16 & 19 & 20 & 27 & 28 \\
12 & 1 & 4 & 6 & 8 & 9 & 11 & 13 & 21 & 22 & 23 & 27 & 28 \\
13 & 1 & 5 & 7 & 8 & 10 & 11 & 12 & 21 & 24 & 25 & 27 & 28 \\
14 & 2 & 3 & 4 & 5 & 15 & 16 & 17 & 19 & 21 & 22 & 24 & 27 \\
15 & 2 & 3 & 4 & 5 & 14 & 16 & 18 & 20 & 21 & 23 & 25 & 28 \\
16 & 2 & 3 & 8 & 11 & 14 & 15 & 17 & 18 & 19 & 20 & 27 & 28 \\
17 & 2 & 6 & 7 & 8 & 14 & 16 & 18 & 19 & 22 & 24 & 26 & 27 \\
18 & 2 & 6 & 7 & 8 & 15 & 16 & 17 & 20 & 23 & 25 & 26 & 28 \\
19 & 3 & 9 & 10 & 11 & 14 & 16 & 17 & 20 & 22 & 24 & 26 & 27 \\
20 & 3 & 9 & 10 & 11 & 15 & 16 & 18 & 19 & 23 & 25 & 26 & 28 \\
21 & 4 & 5 & 12 & 13 & 14 & 15 & 22 & 23 & 24 & 25 & 27 & 28 \\
22 & 4 & 6 & 9 & 12 & 14 & 17 & 19 & 21 & 23 & 24 & 26 & 27 \\
23 & 4 & 6 & 9 & 12 & 15 & 18 & 20 & 21 & 22 & 25 & 26 & 28 \\
24 & 5 & 7 & 10 & 13 & 14 & 17 & 19 & 21 & 22 & 25 & 26 & 27 \\
25 & 5 & 7 & 10 & 13 & 15 & 18 & 20 & 21 & 23 & 24 & 26 & 28 \\
26 & 6 & 7 & 9 & 10 & 17 & 18 & 19 & 20 & 22 & 23 & 24 & 25 \\
27 & 8 & 11 & 12 & 13 & 14 & 16 & 17 & 19 & 21 & 22 & 24 & 28 \\
28 & 8 & 11 & 12 & 13 & 15 & 16 & 18 & 20 & 21 & 23 & 25 & 27
\end{tabular}

EXCEPTIONAL GRAPH II:

\begin{tabular}{|c|c|c|c|c|c|c|c|c|c|c|c|c|}
\hline vertex & & & & its & Ive & ghb & & & & & & \\
\hline 1 & 2 & 3 & 4 & 5 & 6 & 7 & 8 & 9 & 10 & 11 & 12 & 13 \\
\hline 2 & 1 & 3 & 4 & 5 & 6 & 7 & 8 & 14 & 15 & 16 & 17 & 18 \\
\hline 3 & 1 & 2 & 4 & 5 & 9 & 10 & 11 & 14 & 15 & 16 & 19 & 20 \\
\hline 4 & 1 & 2 & 3 & 6 & 9 & 12 & 13 & 14 & 15 & 16 & 21 & 22 \\
\hline 5 & 1 & 2 & 3 & 7 & 8 & 10 & 11 & 14 & 17 & 19 & 23 & 24 \\
\hline 6 & 1 & 2 & 4 & 7 & 8 & 12 & 13 & 15 & 18 & 21 & 25 & 26 \\
\hline 7 & 1 & 2 & 5 & 6 & 8 & 10 & 12 & 17 & 18 & 23 & 25 & 27 \\
\hline 8 & 1 & 2 & 5 & 6 & 7 & 11 & 13 & 17 & 18 & 24 & 26 & 28 \\
\hline 9 & 1 & 3 & 4 & 10 & 11 & 12 & 13 & 16 & 20 & 22 & 27 & 28 \\
\hline 10 & 1 & 3 & 5 & 7 & 9 & 11 & 12 & 19 & 20 & 23 & 25 & 27 \\
\hline 11 & 1 & 3 & 5 & 8 & 9 & 10 & 13 & 19 & 20 & 24 & 26 & 28 \\
\hline 12 & 1 & 4 & 6 & 7 & 9 & 10 & 13 & 21 & 22 & 23 & 25 & 27 \\
\hline 13 & 1 & 4 & 6 & 8 & 9 & 11 & 12 & 21 & 22 & 24 & 26 & 28 \\
\hline 14 & 2 & 3 & 4 & 5 & 15 & 16 & 17 & 19 & 21 & 22 & 23 & 24 \\
\hline 15 & 2 & 3 & 4 & 6 & 14 & 16 & 18 & 19 & 20 & 21 & 25 & 26 \\
\hline 16 & 2 & 3 & 4 & 9 & 14 & 15 & 17 & 18 & 20 & 22 & 27 & 28 \\
\hline 17 & 2 & 5 & 7 & 8 & 14 & 16 & 18 & 22 & 23 & 24 & 27 & 28 \\
\hline 18 & 2 & 6 & 7 & 8 & 15 & 16 & 17 & 20 & 25 & 26 & 27 & 28 \\
\hline 19 & 3 & 5 & 10 & 11 & 14 & 15 & 20 & 21 & 23 & 24 & 25 & 26 \\
\hline 20 & 3 & 9 & 10 & 11 & 15 & 16 & 18 & 19 & 25 & 26 & 27 & 28 \\
\hline 21 & 4 & 6 & 12 & 13 & 14 & 15 & 19 & 22 & 23 & 24 & 25 & 26 \\
\hline 22 & 4 & 9 & 12 & 13 & 14 & 16 & 17 & 21 & 23 & 24 & 27 & 28 \\
\hline 23 & 5 & 7 & 10 & 12 & 14 & 17 & 19 & 21 & 22 & 24 & 25 & 27 \\
\hline 24 & 5 & 8 & 11 & 13 & 14 & 17 & 19 & 21 & 22 & 23 & 26 & 28 \\
\hline 25 & 6 & 7 & 10 & 12 & 15 & 18 & 19 & 20 & 21 & 23 & 26 & 27 \\
\hline 26 & 6 & 8 & 11 & 13 & 15 & 18 & 19 & 20 & 21 & 24 & 25 & 28 \\
\hline 27 & 7 & 9 & 10 & 12 & 16 & 17 & 18 & 20 & 22 & 23 & 25 & 28 \\
\hline 28 & 8 & 9 & 11 & 13 & 16 & 17 & 18 & 20 & 22 & 24 & 26 & 27 \\
\hline
\end{tabular}




\section{EXCEPTIONAL GRAPH III:}

Applying the permutation $(3,7)(4,8)(10,12,15)(9,17,14,18,16,13)$ $(22,24,28)(19,23,26,21,27,20,25)$ to Chang's original numbering of the vertices, we obtain a more economical description, with the vertices 3 to 8 and 19 to 28 having the same sets of neighbours as in graph II. For the twelve remaining vertices, the situation is as follows:

\begin{tabular}{c|rrrrrrrrrrrr} 
vertex & \multicolumn{10}{|c}{ its twelve neighbours } \\
\hline 1 & 2 & 3 & 4 & 5 & 6 & 7 & 8 & 9 & 11 & 12 & 15 & 17 \\
2 & 1 & 3 & 4 & 5 & 6 & 7 & 8 & 10 & 13 & 14 & 16 & 18 \\
9 & 1 & 3 & 4 & 11 & 12 & 15 & 16 & 17 & 20 & 22 & 27 & 28 \\
10 & 2 & 3 & 5 & 7 & 14 & 16 & 18 & 19 & 20 & 23 & 25 & 27 \\
11 & 1 & 3 & 5 & 8 & 9 & 15 & 17 & 19 & 20 & 24 & 26 & 28 \\
12 & 1 & 4 & 6 & 7 & 9 & 15 & 17 & 21 & 22 & 23 & 25 & 27 \\
13 & 2 & 4 & 6 & 8 & 14 & 16 & 18 & 21 & 22 & 24 & 26 & 28 \\
14 & 2 & 3 & 4 & 5 & 10 & 13 & 16 & 19 & 21 & 22 & 23 & 24 \\
15 & 1 & 3 & 4 & 6 & 9 & 11 & 12 & 19 & 20 & 21 & 25 & 26 \\
16 & 2 & 3 & 4 & 9 & 10 & 13 & 14 & 18 & 20 & 22 & 27 & 28 \\
17 & 1 & 5 & 7 & 8 & 9 & 11 & 12 & 22 & 23 & 24 & 27 & 28 \\
18 & 2 & 6 & 7 & 8 & 10 & 13 & 16 & 20 & 25 & 26 & 27 & 28
\end{tabular}

The proof of Theorem 1 in Section 4 will be based on the fact that these three exceptional graphs contain subgraphs that are not realizable in our geometric context. In exceptional graph I, the vertices 13 and 28 are adjacent; their six common neighbours induce the subgraph shown in Fig. 1. In exceptional graphs II and III, the vertices 1 and 2 are adjacent; their six common neighbours induce the subgraph shown in Fig. 2. Moreover, the
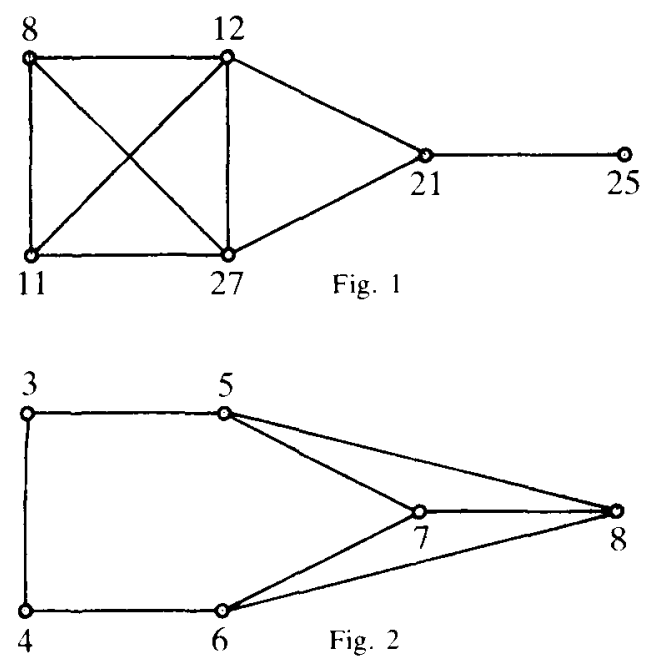


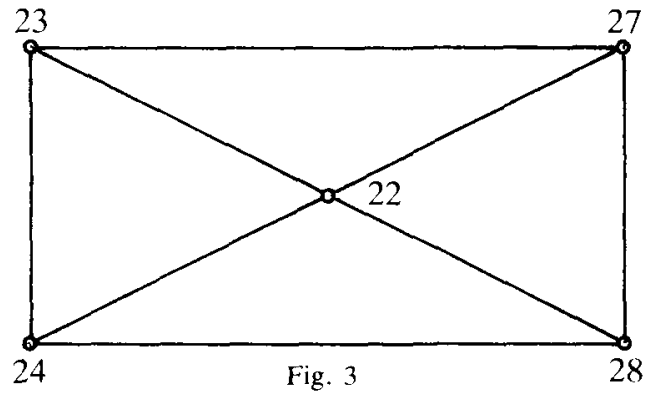

vertices $22,23,24,27,28$ induce the subgraph shown in Fig. 3. We shall also need the fact that, among the vertices 1 to 8 , only 4 is a neighbour of vertex 22 , only 7 one of vertex 27 , only 8 one of vertex 28 , only 5 and 7 are neighbours of vertex 23 and only 5 and 8 neighbours of vertex 24 .

\section{Prerequisites from linear geometry}

The following results $\mathrm{P} 1$ to $\mathrm{P} 5$ are basic and well-known properties of FLS's; for proofs, see - for instance - de Witte (1975a). Both P6 and the embedding result, mentioned in Section 1 and of which P7 and P8 are particular cases, will be found in de Witte (1975b). A good idea about the way to prove them can be obtained from Section V.1 of Totten (1974). The embedding result has also been found by Vanstone (1974), who announced it shortly after a first and weaker version of de Witte (1975b) was circulated under the title 'On the embeddability of restricted linear spaces' (Waterloo, April-May 1973). The two lemmas have been "distilled" from Bose-Shrikhande (1973): redundant assumptions have been cut out and the proofs have been adapted accordingly.

P1. For all $u_{\alpha}$, we have $p=1+\Sigma_{\sigma} r_{c \alpha}\left(a_{s}-1\right)$.

P2. Any line $x_{\sigma}$ meets $1+\sum_{\alpha} r_{\sigma u}\left(b_{\alpha}-1\right)$ lines, itself included.

P3. If $u_{\alpha}$ lies outside $x_{\sigma}$, then $b_{\alpha}-a_{\sigma}$ counts the number of lines passing through $u_{\alpha}$ and missing $x_{\sigma}$. Hence, if $b_{1}=n+1 \geqq 2$, then $a_{1} \leqq n+1$ and any $(n+1)$-line meets every (other) line.

P4. If $b_{1}=a_{1}=n+1$, then $q \leqq n^{2}+n+1$ and equality holds iff all points are of the same degree.

P5. If $a_{\sigma}=a$ for all $x_{\alpha}$, then $b_{\alpha}=b$ for all $u_{\alpha}$, with $b=(p-1) /(a-1)$. Moreover, if $b=a+m$, then $a$ divides $m(m-1)$. In particular, if $m=2$, then $a=2$ and $p=5$, i.e. the FLS is the complete graph on five points. 
P6. If $b_{\alpha}=n+1$ for all $u_{\alpha}, q=n^{2}+n+1$ and $p=n^{2}-1$, then $\Sigma_{\sigma}\left(a_{\sigma}-n\right)^{2}=q$.

P7. Any FAP is embeddable in an FPP of the same order.

P8. Any non-trivial FLS such that $p=n^{2}, b_{\alpha}=n+1$ for all $u_{\alpha}$ and $a_{1}=a_{2}=n+1$ is embeddable in an FPP of order $n(\geqq 3)$.

Lemma 1. In any FLS with $q=n^{2}+n+1$ and $b_{\alpha}=n+1$ for all $u_{\alpha}$, the following properties hold:

(1) any $(n-1)$-line misses $2 n$ lines;

(2) if two (n-1)-lines miss each other, then there are $n$ lines missing them both;

(3) if two distinct $(n-1)$-lines meet each other, then there are four lines missing them both.

Proof. By P2, any $(n-1)$-line $x_{\sigma}$ misses

$$
q-1-\sum_{\alpha} r_{\sigma \alpha}\left(b_{\alpha}-1\right)=n^{2}+n-n a_{\sigma}=2 n
$$

lines. Next, let the $(n-1)$-lines $x$ and $y$ miss each other. By P3, through each point of $x$ there is still one other line missing $y$. Hence, of the $2 n$ lines missing $y$ there are $n$ that meet $x$ and so $n$ that miss $x$. Finally, let the distinct $(n-1)$-lines $x$ and $y$ both pass through $w$. By P3, through each point $\neq w$ of $x$ there are two lines missing $y$. Hence, of the $2 n$ lines missing $y$ there are $2(n-2)$ that meet $x$ and so four that miss $x$. Q.E.D.

Note. If $a_{r}=n \pm 1$ for all $x_{\sigma}$, then P3 implies that all the missing lines mentioned in Lemma 1 are of degree $n-1$.

LEMMA 2. Let $\mathscr{L}$ be an FLS with $b_{\alpha}=n+1$ for all $u_{\alpha}, a_{\sigma}=n \pm 1$ for all $x_{\sigma}$, and with the graph defined on the set of $(n-1)$-lines by the disjointness relation equal to the line graph of the complete graph $K_{s}(s \geqq 2)$. Then $\mathscr{L}$ is embeddable in an FPP of even order $n(\geqq 4)$. Moreover, $s=n+2$ and $p=n^{2}-1$.

Proof. Since $s \geqq 2$, the line graph of $K_{s}$ is nonempty. Hence there are $(n-1)$-lines, and so $n$ is an integer $\geqq 3$. It is then clear that there is a bijection between the $(n-1)$-lines and the pairs $\{i, j\}$ where $i$ and $j$ are distinct integers from 1 to $s$, in such a manner that two $(n-1)$-lines are disjoint iff the corresponding pairs have one integer in common.

Then $\mathscr{L}$ can be extended as follows. For each integer $i$ from 1 to $s$, a new point $u_{p+i}$ is introduced, and to each $(n-1)$-line two new points are added, namely $u_{p+i}$ and $u_{p+j}$ to the line (corresponding to the pair) $\{i, j\}$. The 
$(n+1)$-lines are left untouched, and no new lines are introduced. We claim that the resulting structure $\mathscr{L}^{*}$ is an FPP of order $n$. Clearly all its lines are of

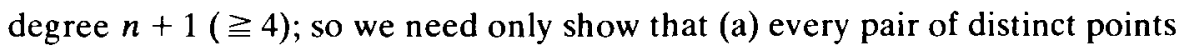
is included in at least one line and that (b) every two distinct lines have precisely one point in common.

(a) This requires proof only if at least one of the points is new. If both are new, $u_{p+i}$ and $u_{p+j}$ say, then they belong to the extended line $\{i, j\}$. If one is old and one new, $u_{\alpha}$ and $u_{p+i}$ say, and $u_{\alpha}$ did not lie on the $(n-1)$-line $\{i, j\}$, then we know from $\mathrm{P} 3$ that there were two $(n-1)$-lines through $u_{\alpha}$ that missed the line $\{i, j\}$. They correspond to pairs $\{i, k\}$ and $\left\{j, k^{\prime}\right\}$ with $k \neq k^{\prime}$. Clearly both $u_{\alpha}$ and $u_{p+i}$ belong to the extended line $\{i, k\}$.

(b) If at least one of the lines was an $(n+1)$-line in $\mathscr{L}$, then it met every other line in one point of $\mathscr{L}$. Now no new points have been added to it. If both were $(n-1)$-lines in $\mathscr{L}$, corresponding to $\{i, j\}$ and $\left\{i^{\prime}, k\right\}$ say, then consider $E=\{i, j\} \cap\left\{i^{\prime}, k\right\}$. If $E$ is empty, then the lines $\{i, j\}$ and $\left\{i^{\prime}, k\right\}$ met in one point of $\mathscr{L}$ and their extensions cannot meet in any new point. If $E$ is a singleton, say $i=i^{\prime}$, then the lines $\{i, j\}$ and $\{i, k\}$ were disjoint in $\mathscr{L}$ and their extensions meet in one new point, namely $u_{p+i}$.

Hence $\mathscr{L}$ is an FLS with $p=n^{2}+n+1-s$ points. Now the $n+1$ lines of $\mathscr{L}^{*}$ passing through $u_{p+s}$ must all be extensions of $(n-1)$-lines in $\mathscr{L}$ and all new points $\neq u_{p+s}$ must lie on precisely one of them; so $n+1=s-1$ and $p=n^{2}-1$.

Finally, it will follow from Lemma 3 that the number of $(n+1)$-lines through any point equals $n / 2$; so $n$ must be even. Q.E.D.

\section{Results and proofs}

THEOREM 1. Let $\mathscr{L}$ be an FLS satisfying the Bose-Shrikhande conditions (a) (b) (c). Then $\mathscr{L}$ is embeddable in an FPP of even order $n(\geqq 4)$.

Proof. It is not difficult to see that $n$ must be an even integer $\geqq 3$. In view of Lemma 1, the note following it, and the assumption about the number of $(n-1)$-lines, all four conditions of the characterization theorem in Section 2 are satisfied for $m=n+2$ by the graph $G$ defined on the set of $(n-1)$-lines by the disjointness relation. If $G$ is the line graph of the complete graph $K_{n+2}$, then Lemma 2 implies that $\mathscr{L}$ is embeddable in an FPP of order $n$. If $G$ is not the line graph of $K_{n+2}$, then $n=6$ and $G$ is one of the three exceptional graphs. We must prove that this is impossible.

1. Since $n=6, \mathscr{L}$ has thirty-five points, any 7 -line meets every (other) line, and there are three 7-lines and four 5-lines through any point of $\mathscr{L}$. This 
implies that, if $x$ and $y$ are disjoint 5-lines and $u$ is a point lying outside both $x$ and $y$, then the four 5-lines through $u$ show one of the following patterns:

(a) two meet both $x$ and $y$, and two miss both $x$ and $y$;

(b) one meets both $x$ and $y$, one misses both $x$ and $y$, one meets $x$ and misses $y$, and one misses $x$ and meets $y$.

(c) two meet $x$ and miss $y$, and two miss $x$ and meet $y$.

From this it follows that, if points $u$ and $v$ show patterns (a) and (c) respectively, then the line $u v$ cannot be a 5 -line.

2. With respect to any set $S$ of 5 -lines all parallel to at least one 5 -line, the weight of a point will be defined as the number of lines in $S$ passing through it. Since no three lines in $S$ can pass through the same point (necessarily of degree seven), the weight of a point will equal 0,1 or 2 . We shall talk of null points, single points and double points respectively. Denoting the number of lines in $S$ by $s$, the number of pairs of disjoint lines in $S$ by $t$, and the number of points of weight $i$ by $p_{i}(i=0,1,2)$, we have

$$
\begin{aligned}
& p_{0}+p_{1}+p_{2}=p=35, \\
& p_{1}+2 p_{2}=5 s, \\
& p_{2}+t=s(s-1) / 2 .
\end{aligned}
$$

Hence

$$
s(s-1) / 2-t=p_{2}=5(s-7)+p_{0} .
$$

It is obvious that $S$ corresponds to a subgraph of $G$ with $s$ vertices and $t$ edges.

Since any 7-line meets every (other) line, the sum of the weights of the points lying on it equals $s$. In particular, if $s=7$, then the number of double points on a 7-line equals the number of null points on it.

3. If $G$ is the exceptional graph $I$, let $S$ be the set of 5-lines corresponding to the vertices $8,11,12,13,21,27,28$. Clearly $s=7, t=1+2.5+8=19$, and the lines in $S$ are all parallel to lines 13 and 28 . So (*) yields $p_{0}=p_{2}=2$. But, for any double point, there must be at least one null point on each of the three 7-lines passing through it. So $p_{2} \geqq 1$ implies $p_{0} \geqq 3$. This contradicts $p_{0}=p_{2}=2$.

4. If $G$ is the exceptional graph $I I$ or $I I I$, let $S$ be the set of 5-lines corresponding to the vertices $1,2,3,4,5,6,7,8$. Clearly $s=8, t=$ $1+2.6+8=21$, and the lines in $S$ are all parallel to lines 1 and 2 . So (*) yields $p_{0}=2, p_{2}=7$. Moreover, all points on lines 1 and 2 are single points. With respect to these two disjoint 5 -lines, the double points show pattern (a) and the null points pattern (c). For the null points, this follows from the fact that 
all lines missing both lines 1 and 2 are members of $S$. Any line connecting a double point to a null point must therefore be a 7 -line. Now any 7 -line meets both lines 1 and 2 (in single points). So, counting the sum of the weights of its points, we see that any 7 -line contains either one double point and six single points, or two double points, four single points and one null point, or three double points, two single points and two nuil points. Consequently, the line connecting the two null points must be a 7-line and contain three double points. (Consider how a null point could be joined to the double points.)

It follows from Fig. 2 that the corresponding 5-lines and the seven double points are situated as in Fig. 4, where we have denoted the double points in an obvious fashion by $36,37,38,45,47,48,56$. There are only two ways for a 7 -line to pass through three of these double points: either through $56,37,48$ or through $56,38,47$. Before distinguishing these two cases, we still should notice that a 5 -line not in $S$ and missing at most one line of $S$ (as is the case for lines $22,27,28$ ) must pass through at least two double points. Similarly, if it misses at most two lines of $S$ (as is the case for lines 23,24 ), it must pass through at least one double point.

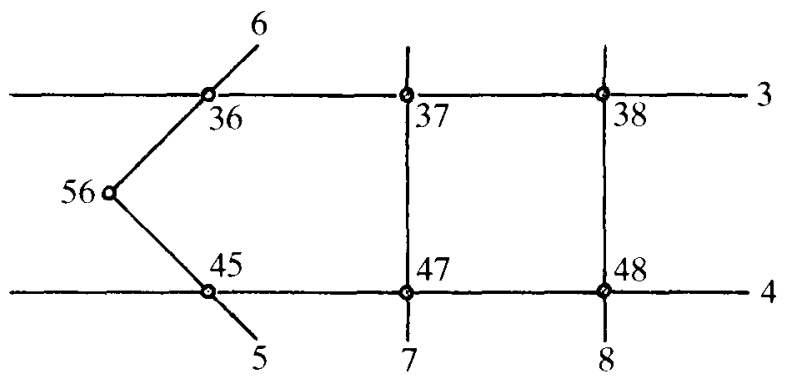

Fig. 4

First, let 56, 37, 48 lie on a 7-line. Since line 22 misses line 4, it must join the double points 56 and 38 . Since line 23 misses lines 5, 7 and 22 , it must pass through 36 or 48 . Since line 27 misses lines 7 and 22, it must pass through 36 and 45 or 48 . But lines 23 and 27 are disjoint; so line 27 joins 36 and 45 . Consider now line 28 , which misses lines 8,22 and 27 . The only double points left for it are 37 and 47, which cannot be used together since they are already joined by line 7 .

To derive a contradiction when $56,38,47$ are on a 7 -line, it suffices to permute the roles of lines 7 and 8,23 and 24 , and 27 and 28 .

This completes the proof of Theorem 1.

LEMMA 3. Let $\mathscr{L}$ be an FLS with $b_{\alpha}=n+1$ for all $u_{\alpha}$ and $a_{\sigma}=n \pm 1$ for all $x_{\sigma}$. Then every point lies on $\left(p-n^{2}+n+1\right) / 2$ lines of degree $n+1$ and on 
$\left.n^{2}+n+1-p\right) / 2$ lines of degree $n-1$. Moreover, if $\mathscr{L}$ has at least one line but is not the complete graph on five points, then $q=n^{2}+n+1$, with $\left.p n+p-n^{3}+1\right) / 2$ lines of degree $n+1$ and $\left(n^{2}+n+1-p\right)(n+1) / 2$ lines of degree $n-1$.

ProOF. Denoting the number of $(n+1)$-lines through $u_{\alpha}$ by $s_{\alpha}(1 \leqq \alpha \leqq$ p), we find from P1 that

$$
p=1+n s_{\alpha}+(n-2)\left(n+1-s_{\alpha}\right),
$$

whence $s_{\alpha}=\left(p-n^{2}+n+1\right) / 2$. Moreover, if $\mathscr{L}$ has at least one line but is not the complete graph on five points, then P5 implies $a_{1}=n+1$. By P4, we get $q=n^{2}+n+1$. Besides, any $(n+1)$-line meets $1+(n+1)\left(s_{\alpha}-1\right)=$ $\left(p n+p-n^{3}+1\right) / 2$ lines of degree $n+1$. By P3, this gives the full number of $(n+1)$-lines. Q.E.D.

Corollary 1. Let $\mathscr{L}$ be an FLS with $b_{\alpha}=n+1$ for all $u_{\alpha}, a_{\sigma}=n \pm 1$ for all $x_{\sigma}$, with at least one line, and with $(n+2)(n+1) / 2$ lines of degree $n-1$. Unless $\mathscr{L}$ is the complete graph on five points, the Bose-Shrikhande conditions (a) (b) (c) all hold and $\mathscr{L}$ is embeddable in an FPP of even order $n(\geqq 4)$.

Proof. If $\mathscr{L}$ is not the complete graph on five points, then the assumptions of Lemma 3 are satisfied. So

$$
(n+2)(n+1) / 2=\left(n^{2}+n+1-p\right)(n+1) / 2,
$$

whence $p=n^{2}-1$. Then everything follows at once from Lemma 3 and Theorem 1 .

Corollary 2. Let $\mathscr{L}$ be an FLS with $p=n^{2}-1, b_{\alpha}=n+1$ for all $u_{\alpha}$ and $a_{1}=n+1$, but without any $n$-line. Then the Bose-Shrikhande conditions $(a)$ (b) (c) all hold and $\mathscr{L}$ is embeddable in an FPP of even order $n$ ( $\geqq 4)$.

Proof. By P4, we have $q=n^{2}+n+1$. Hence P6 yields $\Sigma_{\sigma}\left(a_{\sigma}-n\right)^{2}=q$. Since there is no $n$-line, this implies $\left(a_{\sigma}-n\right)^{2}=1$ i.e. $a_{\sigma}=n \pm 1$, for all $x_{r}$. Moreover $p \neq 5$. Then everything follows at once from Lemma 3 and Theorem 1.

LEMMA 4. Let $\boldsymbol{n}$ be an integer and $\mathscr{L}$ a non-trivial FLS such that $p \geqq n^{2}-1, b_{1} \leqq n+1$ and $a_{1} \leqq n$. Then $p \leqq n^{2}$ and $\mathscr{L}$ is either an FAP of order $n$ or a punctured FAP of order $n$ (i.e. with one point removed).

Proof. Clearly $n \geqq 2$. By P1, we have

$$
\begin{aligned}
n^{2}-1 & \leqq p=1+\sum_{\sigma} r_{\sigma \alpha}\left(a_{\sigma}-1\right) \\
& \leqq 1+\left(a_{1}-1\right) b_{\alpha} \leqq 1+(n-1)(n+1)=n^{2}
\end{aligned}
$$


If $p=n^{2}$, all inequalities are flattened out to equalities, yielding $a_{\sigma}=b_{\alpha}-1=$ $n$ for all $x_{\sigma}$ and $u_{\alpha}$. Hence $\mathscr{L}$ is an FAP of order $n$. If $p=n^{2}-1$, the result is obvious for $n=2$. So suppose $n \geqq 3$. If $b_{\alpha} \leqq n$, the above formula would imply $n^{2}-1 \leqq 1+(n-1) n$, and so $n \leqq 2$. Hence all points are of degree $n+1$. Denoting the number of $n$-lines through $u_{\alpha}$ by $s_{\alpha}$ and the number of $(n-1)$-lines through $u_{\alpha}$ by $t_{\alpha}$, we find from P1 that

$$
\begin{aligned}
n^{2}-1 & \leqq 1+(n-1) s_{\alpha}+(n-2) t_{\alpha}+(n-3)\left(n+1-s_{\alpha}-t_{\alpha}\right) \\
& =2 s_{\alpha}+t_{\alpha}+n^{2}-2 n-2,
\end{aligned}
$$

whence $2 n+1 \leqq 2 s_{\alpha}+t_{\alpha} \leqq s_{\alpha}+n+1$, and so $s_{\alpha} \geqq n$. Now $s_{\alpha}=n+1$ would imply $p=1+(n-1)(n+1)=n^{2}$, which is false. Hence $s_{\alpha}=n$, and so $t_{\alpha}=1$. This ensures that there are no lines of degree $\leqq n-2$ and that the $(n-1)$-lines partition the points of $\mathscr{L}$. Hence the number of $(n-1)$-lines equals $p /(n-1)=n+1$. Introducing one new point and adding it to each of these $(n-1)$-lines, we obtain an FLS, as can be easily verified. All its lines are of degree $n$ and all its points are of degree $n+1$. So it is an FAP of order $n$. Clearly $\mathscr{L}$ can be obtained from it by removing one point. Q.E.D.

Corollary 3. A non-trivial FLS is an affine plane iff both $\left(b_{1}-1\right)^{2} \leqq p$ and $a_{1}^{2} \leqq p$ hold.

Proof. In one direction, the result is obvious. To prove the other direction, it suffices to define $n$ as the integral part of the positive square root of $p$ and to apply Lemma 4 .

NotE. Other characterizations of FAP's have recently been given in de Witte (1974) and Totten (1975).

COROLLARY 4. Let $n$ be an integer and $\mathscr{L}$ an FLS such that $p \geqq n^{2}-1$ and $b_{1} \leqq n+1$. Then $\mathscr{L}$ satisfies $q \leqq n^{2}+n+1$.

PRoOF. The result is trivial if $q \leqq 1$. So let $\mathscr{L}$ be non-trivial. If $a_{1}=n+1$, the result follows from P4. Otherwise $a_{1} \leqq n$ and Lemma 4 applies, whence $q \leqq n^{2}+n$. Q.E.D.

THEOREM 2. Let $n$ be an integer and $\mathscr{L}$ a non-trivial FLS such that $p=n^{2}-1$ and $b_{1} \leqq n+1$. Then $\mathscr{L}$ is embeddable in an FPP of order $n$.

Proof. If $a_{1} \leqq n$, the result follows at once combining Lemma 4 and P7. So let $a_{1}=n+1$. Clearly $n^{2}-1=p \geqq a_{1}+1=n+2$, whence $n \geqq 3$.

First, let there be at least one $n$-line. It will be convenient to call a point special if its degree $\neq n+1$. P3 implies that all special points lie on $x_{1}$. Let $t$ denote the number of special points. We claim that $t \geqq 2$ is impossible for 
$n \geqq 4$. For it follows from P3 that $t \geqq 2$ implies $a_{2}=n$. Applying P1 at any special point, we obtain $n^{2}-1 \leqq 1+n+(n-1)^{2}$, and so $n \leqq 3$.

We now claim that there exists an $n$-line meeting $x_{1}$ in an $(n+1)$-point. Suppose not. Then P3 would imply $t \geqq 1$. By P3 again, no $(n+1)$-point on $x_{1}$ zould therefore lie on another $(n+1)$-line. But by hypothesis the $(n+1)$ points on $x_{1}$ lie on no $n$-lines either. So P1 yields $n^{2}-1 \leqq 1+n+(n-2) n$, whence $n \leqq 2$. This contradiction implies $t=n+1 \geqq 4$. As we have seen, this zannot happen if $n \geqq 4$. So let $n=3$. Clearly no special point is of degree two. Hence P2 yields $q=1+2.4=p+1$. It follows from the work of Bridges (1972) that no such FLS exists; see also Section VI.1 of Totten (1974). Though here is no need to appeal to such a high-powered result! For clearly all eight ines $\neq x_{1}$ are of degree three. So removing the four points of $x_{1}$, we would be left with an FLS consisting of four points and eight 2-lines, which is plainly mpossible. This completes the proof of our second claim.

Consequently, all special points are of degree $n$. Let $x_{\sigma}$ be an $n$-line neeting $x_{1}$ in an $(n+1)$-point. Through any $(n+1)$-point on $x_{1}$ there is orecisely one line parallel to $x_{\sigma}$, and it follows from P3 that any such parallel neets $x_{1}$ in an $(n+1)$-point. No two such parallels can meet since their point of intersection would be of degree $\geqq n+2$. We introduce one new point, $u^{*}$ ;ay, and add it to each of these parallels. Moreover, for each $n$-point $u_{\alpha}$ on $x_{1}$ we introduce the 2-line $u_{\alpha} u^{*}$-althogether $t$ of them. We thus obtain an FLS, $\mathscr{L}^{*}$ say, as can be easily verified. All its $n^{2}$ points are of degree $n+1$, and it las at least two $(n+1)$-lines, namely $x_{1}$ and $x_{\sigma} \cup\left\{u^{*}\right\}$. By P8, $\mathscr{L}^{*}$ is zmbeddable in an FPP of order $n$. Therefore so is $\mathscr{L}$.

Next, let there be no $n$-lines in $\mathscr{L}$. Then all points are of degree $n+1$. For therwise all lines outside $u_{p}$ would be of degree $\leqq n-1$. At any other point $u_{\alpha}$, P1 would therefore yield $n^{2}-1 \leqq 1+n+(n-2) n$, whence $n \leqq 2$. Hence Corollary 2 applies, and so $\mathscr{L}$ is embeddable in an FPP of even order $n(\geqq 4)$.

This completes the proof of Theorem 2.

Note. It is possible to get additional information about $\mathscr{L}$ when it has soth an $(n+1)$-line and an $n$-line. Note that $q+t=n^{2}+n+1$, and so $l^{-} p=n+2-t$. We have already seen that $t \leqq 1$ if $n \geqq 4$ and that $t \neq 4$ if $\imath=3$. Once the embedding is obtained, it is not hard to exclude both $t=0$ and $: \geqq 3$ if $n=3$. The remaining values are all possible: $t$ assumes the values 0 and 1 if $n \geqq 4$ and the values 1 and 2 if $n=3$. For it is obvious that, given any FPP of order $n \geqq 4$, it is possible to select and remove $n+2$ points so as to sbtain FLS's of the above type with $t=0$ and with $t=1$. Applying these :onstructions in an FPP of order three, we find examples with $t=1$ and with $:=2$. For $t=1$, remove five points forming an FLS with $a_{1}=3, a_{2}=2$. The 
resulting FLS can be described in a simple intrinsic manner, making its unicity (up to isomorphism) quite plain. For $t=2$, remove five points forming an FLS with $a_{1}=a_{2}=3$. The resulting FLS has been shown to be unique (up to isomorphism) in Section VI.3 of Totten (1974).

\section{References}

R. C. Bose (1947), 'Mathematical theory of the symmetrical factorical design', Sankhyã 8 107-166.

R. C. Bose (1973), 'Graphs and designs', pp. 1-104 of: Finite geometric structures and thei applications, Centro Internazionale Matematico Estivo, Bressanone, June 1972 (Edi. zioni Cremonese, Roma).

R. C. Bose and S. S. Shrikhande (1973), 'Embedding the complement of an oval in a projective plane of even order', Discrete Math. 6, 305-312.

Marc Bouten and Paul de Witte (1965), 'A new proof of an inequality of Szekeres. de Bruijn and Erdös', Bull. Soc. Math. Belg. 17, 475-483.

W. G. Bridges (1972), 'Near 1-designs', J. Combinatorial Theory (A) 13, 116-126.

Chang Li-chien (1959), 'The uniqueness and nonuniqueness of the triangular association schemes', Science Record (Peking), Math. New Ser. 3, 604-613.

Chang Li-chien (1960), 'Association schemes of partially balanced designs with parameters $v=28, n_{1}=12, n_{2}=15$ and $p_{11}^{2}=4$ ', Science Record (Peking), Math. New Ser 4, 12-18.

Frank Harary (1969), Graph theory (Addison-Wesley, Reading).

B. Qvist (1952), 'Some remarks concerning curves of the second degree in a finite plane', Ann Acad. Sci. Fenn. Ser. A 1, no. 134.

James E. Totten (1974), Classification of restricted linear spaces, Doctoral dissertation, University of Waterloo.

Jim Totten (1975), 'Basic properties of restricted linear spaces', Discrete Math. 13, 67-74.

Scott A. Vanstone (1974), The structure of regular pair-wise balanced designs, Doctoral dissertation, University of Waterloo.

Paul de Witte (1974), 'Restricted linear spaces with a square number of points', Simon Stevin 48. $107-120$.

Paul de Witte (1975a), 'Combinatorial properties of finite linear spaces II', Bull. Soc. Math. Belg 27. 115-155.

Paul de Witte (1975b), 'On the embeddability of linear spaces in projective planes of order $n$ ', tc appear.

Department of Pure Mathematics,

University of Waterloo,

Waterloo, Ontario, N2L 3G1,

Canada.

Author's current address: Westerkim, 122 Capucienenlaan, B-9300 Aalst, Belgium. 